\title{
KRAS mutations in Slovene patients with colorectal cancer: frequency, distribution and correlation with the response to treatment
}

\author{
ALENKA LICAR ${ }^{1}$, PETRA CERKOVNIK $^{1}$, JANJA OCVIRK $^{2}$ and SRDJAN NOVAKOVIC ${ }^{1}$ \\ Departments of ${ }^{1}$ Molecular Diagnostics and ${ }^{2}$ Medical Oncology, \\ Institute of Oncology Ljubljana, Zaloska 2, 1000 Ljubljana, Slovenia
}

Received December 23, 2009; Accepted February 3, 2010

DOI: 10.3892/ijo_00000596

\begin{abstract}
KRAS mutations are proved as a predictor of response to EGFR-targeted therapies for patients with metastatic colorectal cancer. For identifying the wild-type KRAS (wt-KRAS) responder subset of patients who will benefit from novel agents our laboratory has introduced the TheraSreen K-RAS Mutation $\mathrm{Kit}^{\circledR}$ an allele-specific RT-PCR based assay. Our aim is to describe the validation procedure of this method in our laboratory, determine the portion of colorectal cancer patients with wt-KRAS status, and assess the prognostic power of mutational status for the anti-EGFR therapy outcome in colorectal cancer patients. In this study 302 samples from 273 patients with metastatic colorectal cancer were tested for 7 most common mutations on codon 12 and 13 of the KRAS gene. We used HT-29 and CCL-247 cell lines to determine the sensitivity of the method for different proportions of tumor cells in the sample. We determined that $2 \%$ of cells carrying a KRAS mutation must be present in the sample for an undisputable detection of mutated signal using the LightCycler Adapt Software. Among the tested patients $54.5 \%$ had a wt-KRAS genotype and $45.5 \%$ had a mutated KRAS genotype. The p.Gly12Asp was the most common detected mutation $(38.5 \%)$. Among the cetuximab therapy responders, $85.7 \%$ had a wt-KRAS genotype. We have shown that the RT-PCR method introduced to discriminate between anti-EGFR therapy responders and non-responders is efficient, reliable and quickly applicable. The ratio of mutated versus wt-KRAS patients in our study is similar to ratios reported by other authors, as is the high correlation between wt-KRAS genotype and response to cetuximab therapy. Nevertheless the selection of patients for treatment solely on the basis of KRAS status is not perfect due to the fact that some responders are among the patients with mutated KRAS and some non-responders among the wtKRAS patients.
\end{abstract}

Correspondence to: Professor Srdjan Novakovic, Institute of Oncology Ljubljana, Zaloska 2, 1000 Ljubljana, Slovenia

E-mail: snovakovic@onko-i.si

Key words: colorectal cancer, metastatic, KRAS, mutation

\section{Inroduction}

The epidermal growth factor receptor (EGFR) is a transmembrane tyrosine kinase receptor. It is expressed in epithelial tissues and acts as a cell growth promoter. The EGFR activates at least three major signaling pathways: the RASMAPK, the P13K-Akt and the STAT pathway (1). According to the literature the EGFR contributes to the development and progression of several types of cancer, cancer of the head and neck, ovarian, cervical, bladder, esophageal, gastric, breast, endometrial, lung and colorectal cancer (1). EGFR is overexpressed in 50-80\% of colorectal tumors (2-6) and is therefore a suitable target for anti-cancer therapies. Currently two strategies to attenuate EGFR signaling are in use: monoclonal antibodies that bind to the ligand-binding domain and inhibit the binding of specific ligand (cetuximab and panitumumab) (7) or small EGFR tyrosine kinase inhibitor molecules that bind to the intracellular domain of EGFR and by competing for binding with ATP inhibiting tyrosine phosphorylation (gefitinib, erlotinib) (8). Nevertheless, the anti-EGFR therapies are only effective in a subset of patients with colorectal cancer (9). To optimize benefits and reduce the risk of anti-EGFR therapies, the EGFR as well as the molecules involved in its signaling pathway have been evaluated as potential markers for predicting therapy outcomes. Recent studies demonstrated that KRAS mutations proved as a predictor of response to EGFR-targeted therapies for patients with metastatic colorectal cancer (10-14).

The KRAS gene encodes the human cellular homolog of a transforming gene isolated from the Kirsten rat sarcoma virus. The product of the gene is a small G-protein that functions downstream of EGFR-induced cell signaling. It belongs to the family of RAS proteins that are GDP/GTP-binding proteins that act as intracellular signal transducers by coupling the signal from the cell surface receptors to intracellular targets $(15,16)$. The human KRAS proto-oncogene is mutated in $30-40 \%$ of colorectal cancers. The most frequent alterations are detected in codons 12 and 13 in exon 2 of the KRAS gene (17-19). The KRAS mutations represent an early event in the development and progression of colorectal cancer (20-22). The protein product of mutated KRAS gene has an increased binding affinity for GTP, causing accumulation in the active GTP-bound state by impairing intrinsic GTPase 
activity and conferring resistance to GTPase-activating proteins (23). The signal transduction therefore no longer runs via EGFR, resulting in the fact that patients with KRAS mutations on codon 12 and 13 have poor response to therapy with anti-EGFR inhibitors.

For identifying the wt-KRAS responder subset of patients who will benefit from novel agents targeting the EGFR, a suitable tool for detecting KRAS mutations is required in a laboratory. Our laboratory has introduced a CE-marked TheraScreen K-RAS mutation kit (Roche Applied Science, Mannheim, Germany); an allele-specific RT-PCR based assay. This kit has been approved as in vitro diagnostic device by the EMEA (European Medicines Agency) (24).

Therefore, the objectives of this article are 1) to describe the validation procedure of the TheraScreen method in our laboratory, 2) to determine the portion of colorectal cancer patients with wt-KRAS status, and 3) to assess the power of mutational status for prognostication of the anti-EGFR therapy outcomes in colorectal cancer patients.

\section{Materials and methods}

Patients and tumor samples. In this study 302 samples from 273 patients with metastatic colorectal cancer, treated at the Institute of Oncology Ljubljana from August 2008 to September 2009, were tested for diagnostic purpose. Apart from tumor samples, in some cases, the metastatic tissue samples from the patients were also obtained, with written consent, and tested.

The appropriate formalin-fixed paraffin-embedded tumor tissue block was selected by the appointed pathologist, who also evaluated the percentage of tumor cells in the paraffin slides from the first and last HE-stained cut.

For the validation of the TheraScreen method and assessment of power of mutational status for prognostication of the anti-EGFR therapy outcomes we collected samples from 23 patients. For this reason 30 samples were tested altogether: 25 tumor samples (additional tumor sample was available for two patients) and 5 pair matched non-tumor samples. These 23 patients with metastatic colorectal cancer were treated with cetuximab and chemotherapy as the second, third- or further-line treatment after failure of the treatment with irinotecan. Of the 23 patients treated with cetuximab, 11 were male and 12 were female with the age ranging from 33 to 72 years. The patients were regularly followed up once a week for at least three months after the beginning of the therapy with cetuximab. The response to treatment was evaluated according to the RECIST criteria.

Cell lines. The HT-29 are colorectal adenocarcinoma cells that do not harbor mutations in the KRAS gene. They were grown in advanced EMEM supplemented with $10 \%$ FCS at $37^{\circ} \mathrm{C}$ and $5 \% \mathrm{CO}_{2}$. The CCL247 are colorectal carcinoma cells that harbor the p.Gly13Asp mutation of the KRAS gene and were used as a positive control in mutation assays. They were grown in McCoy's 5A-modified medium at $37^{\circ} \mathrm{C}$ and $5 \% \mathrm{CO}_{2}$.

The mononuclear cells were isolated from fresh peripheral blood with Ficoll-Paque Premium (GE Healthcare Bio-Sciences AB, Uppsala, Sweden). The fresh blood sample was diluted with $1 \mathrm{X}$ PBS and layered on the Ficoll-Paque Premium. The tubes were centrifuged at $4000 \mathrm{rpm}$ for $30 \mathrm{~min}$. The layer of mononuclear cells was collected and washed with PBS three times. Series of different cell mixture samples was prepared to mimic the heterogeneous tumor tissue from a patient. The DNA from the cell mixtures was then isolated and RT-PCR with TheraScreen K-RAS Mutation Kit was performed.

DNA extraction. Non-colored paraffin-embedded sample tissue cuts with the thickness of $10 \mu \mathrm{m}$ (approximately $100 \mu \mathrm{m}$ of tumor tissue) were prepared in an eppendorf tube by the pathologist. The tissue was de-paraffinised by washing with Xylol and rehydrated by washing with $100 \%$ ethanol. The tissue was dried to evaporate all ethanol. For the DNA extraction High Pure PCR template preparation kit (Roche) was used according to the manufacturer's protocol. After the isolation DNA concentration was spectrophotometrically measured at 280/260 $\mathrm{nm}$.

KRAS mutation analysis. For determining the 7 most common mutations on codon 12 and 13 of the KRAS gene we used the TheraSreen K-RAS Mutation Kit (Roche Applied Science). The RT-PCR was performed according to the manufacturer's instructions (25) with a modification in the amount of the sample DNA added. For the testing $100 \mathrm{ng}$ of DNA was added per reaction well. The assay was run on the Roche LightCycler 480 real-time PCR System Instrument II (Roche Applied Science). The results were analyzed using the Light Cycler $^{\circledR}$ Adapt Software (Roche Applied Science).

Statistical analysis. The Statistic Online Computational Resource (SOCR) tool was used for statistical analysis. The Pearson's Chi-square test was used to calculate the p-value for association between KRAS mutation and response to cetuximab. The statistical significance was set at $\mathrm{p}<0.05$.

\section{Results}

Validation procedure for the TheraScreen diagnostic method. For establishing the KRAS genotype testing in our laboratory we tested a panel of 30 retrospective samples from 23 patients ( 5 of the tested tumor samples had matching non-tumor samples) diagnosed with metastatic colorectal carcinoma with the TheraSreen K-RAS Mutation Kit. We compared the results of TheraSreen K-RAS Mutation Kit method with the results of pyrosequencing. The pyrosequencing was performed by the appointed reference laboratory at the Institute of Pathology of the LMU Munich.

DNA isolated from the peripheral blood of a healthy individual was used as a negative control and the DNA isolated from the CCL247 colorectal carcinoma cell line carrying the KRAS p.Gly13Asp mutation as a positive control. The DNA from all the samples was successfully extracted and was of suitable quality for PCR amplification. We detected no mutations in the non-tumor samples from the patients. Of the 25 tested tumor samples 12 (48\%) were positive for KRAS mutations. The positive control sample (CCL247 cell line) tested positive for KRAS p.Gly13Asp mutation, while the negative control sample (blood sample from a healthy individual) gave a wt-KRAS result. 
Table I. Results for KRAS genotyping tested with TheraSreen K-RAS Mutation Kit compared with the results of pyrosequencing.

\begin{tabular}{|c|c|c|c|c|}
\hline No. & Sample ID & Sample type & $\begin{array}{c}\text { TheraScreen } \\
\text { Real-time PCR }\end{array}$ & $\begin{array}{c}\text { Pyrosequencing } \\
\text { Amplicon sequencing }\end{array}$ \\
\hline 1 & KR26 & Non-tumor & wt & wt \\
\hline 2 & KR27 & Non-tumor & wt & wt \\
\hline 3 & KR28 & Non-tumor & wt & wt \\
\hline 4 & KR29 & Non-tumor & wt & wt \\
\hline 5 & KR30 & Non-tumor & wt & wt \\
\hline 6 & KR31 & Whole blood & wt & \\
\hline 7 & KR1 & Tumor & wt & wt \\
\hline 8 & $\mathrm{KR} 2$ & Tumor & wt & wt \\
\hline 9 & KR5 & Tumor & wt & wt \\
\hline 10 & KR9 & Tumor & wt & wt \\
\hline 11 & KR10 & Tumor & wt & wt \\
\hline 12 & KR12 & Tumor & wt & wt \\
\hline 13 & KR15 & Tumor & wt & wt \\
\hline 14 & KR16 & Tumor & wt & wt \\
\hline 15 & KR17 & Tumor & wt & wt \\
\hline 16 & KR18 & Tumor & wt & wt \\
\hline 17 & KR21 & Tumor & wt & wt \\
\hline 18 & KR22 & Tumor & wt & wt \\
\hline 19 & KR23 & Tumor & wt & wt \\
\hline 20 & KR20 & Tumor & $12 \mathrm{ARG}$ & $12 \mathrm{ARG}$ \\
\hline 21 & KR3 & Tumor & 12ASP & 12ASP \\
\hline 22 & KR6 & Tumor & 12ASP & 12ASP \\
\hline 23 & KR13 & Tumor & 12ASP & 12ASP \\
\hline 24 & KR14 & Tumor & 12ASP & 12ASP \\
\hline 25 & KR25 & Tumor & 12ASP & 12ASP \\
\hline 26 & KR4 & Tumor & 12SER & 12SER \\
\hline 27 & KR7 & Tumor & 12SER & 12SER \\
\hline 28 & KR8 & Tumor & $12 \mathrm{VAL}$ & $12 \mathrm{VAL}$ \\
\hline 29 & KR11 & Tumor & 13ASP & 13ASP \\
\hline 30 & KR19 & Tumor & 13ASP & 13ASP \\
\hline 31 & KR24 & Tumor & 13ASP & 13ASP \\
\hline 32 & KR32 & Cell line CCL247 & 13ASP & \\
\hline
\end{tabular}

In our laboratory the determined sensitivity and specificity of the RT-PCR with the TheraScreen K-RAS Mutation Kit method is $100 \%$, as we detected no false positive or false negative samples. This was confirmed by pyrosequencing since the data from real-time PCR and pyrosequencing testing is in $100 \%$ concordance for the tested samples (Table I).

According to the manufacturer, TheraSreen K-RAS Mutation Kit is able to detect $1 \%$ of mutated DNA in a background of wt-DNA. With the intention to confirm the given manufacturer's detection limit (and corresponding sensitivity), the method was tested in our laboratory. To set up the minimum percentage of tumor cells carrying the KRAS p.Gly13Asp mutation in a background of non-tumor cells that are required for successful mutation detection, a series of cell mixture samples was prepared to mimic the heterogeneous tumor tissue from a patient (Table II). We determined that at least $2 \%$ of CCL247 cells carrying the p.Gly13Asp mutation must be present in the sample mixture for successful positive detection by the LightCycler Adapt Software. Nevertheless, when we performed the analysis manually, the apparent amplification of mutated DNA started when $0.2 \%$ of CCL247 carrying KRAS p.Gly13Asp mutation was present in the sample mixture. We observed steeper amplification curves as the percentage of tumor cells in the sample increased (Fig. 1).

We also prepared mixtures of DNA isolated from HT-29 (without KRAS mutation) and CCL247 (harboring the p.Gly13Asp mutation in the $K R A S$ gene). In this case also at least $2 \%$ of CCL247 DNA carrying the p.Gly13Asp mutation must be present in the sample for successful positive detection by the LightCycler Adapt Software. 
Table II. Sensitivity of test, determined in samples containing different proportions of mutated cells.

\begin{tabular}{|c|c|c|c|}
\hline $\begin{array}{l}\text { No. of HT-29 } \\
\text { wt KRAS cells }\end{array}$ & $\begin{array}{l}\text { No. of CCL2 } 47 \text { cells } \\
\text { harboring the KRAS } \\
\text { p.Gly13Asp mutation }\end{array}$ & $\begin{array}{c}\text { No. of } \\
\text { mononuclear cells }\end{array}$ & KRAS genotype \\
\hline $1 \times 10^{6} \quad(4.95 \%)$ & $1 \times 10^{4}(0.05 \%)$ & $1.9 \times 10^{7}(95 \%)$ & wt \\
\hline $2 \times 10^{6} \quad(9.9 \%)$ & $2 \times 10^{4} \quad(0.1 \%)$ & $1.8 \times 10^{7}(90 \%)$ & $\mathrm{wt}$ \\
\hline $4 \times 10^{6} \quad(19.8 \%)$ & $4 \times 10^{4} \quad(0.2 \%)$ & $1.6 \times 10^{7}(80 \%)$ & wt \\
\hline $8 \times 10^{6} \quad(39.6 \%)$ & $8 \times 10^{4} \quad(0.4 \%)$ & $1.2 \times 10^{7}(60 \%)$ & wt \\
\hline $10 \times 10^{6}(49.5 \%)$ & $10 \times 10^{4}(0.5 \%)$ & $1 \times 10^{7}(50 \%)$ & wt \\
\hline $12 \times 10^{6}(59.4 \%)$ & $12 \times 10^{4}(0.6 \%)$ & $0.8 \times 10^{7}(40 \%)$ & wt \\
\hline $14 \times 10^{6}(69.7 \%)$ & $14 \times 10^{4}(0.7 \%)$ & $0.6 \times 10^{7}(30 \%)$ & wt \\
\hline $16 \times 10^{6}(79.2 \%)$ & $16 \times 10^{4}(0.8 \%)$ & $0.4 \times 10^{7}(20 \%)$ & wt \\
\hline $18 \times 10^{6}(89.1 \%)$ & $18 \times 10^{4}(0.9 \%)$ & $0.2 \times 10^{7}(10 \%)$ & $\mathrm{wt}$ \\
\hline $20 \times 10^{6} \quad(99 \%)$ & $20 \times 10^{4} \quad(1 \%)$ & 1 & wt \\
\hline $1.96 \times 10^{6}(98 \%)$ & $4 \times 10^{4} \quad(2 \%)$ & l & p.Gly13Asp \\
\hline $1.94 \times 10^{6}(97 \%)$ & $6 \times 10^{4} \quad(3 \%)$ & l & p.Gly13Asp \\
\hline $1.9 \times 10^{6} \quad(95 \%)$ & $10 \times 10^{4} \quad(5 \%)$ & l & p.Gly13Asp \\
\hline $1.8 \times 10^{6} \quad(90 \%)$ & $20 \times 10^{4} \quad(10 \%)$ & I & p.Gly13Asp \\
\hline $1.6 \times 10^{6} \quad(80 \%)$ & $40 \times 10^{4} \quad(20 \%)$ & l & p.Gly13Asp \\
\hline $1.4 \times 10^{6} \quad(70 \%)$ & $60 \times 10^{4} \quad(30 \%)$ & / & p.Gly13Asp \\
\hline
\end{tabular}

HT-29 colorectal adenocarcinoma cell line with no KRAS mutation, CCL247 colorectal carcinoma cell line carrying KRAS p.Gly13Asp mutation and mononuclear cells isolated from the peripheral blood of a healthy individual were mixed in appropriate volumes. The range of mutated cells was $0.05-30 \%$. The total amount of cells in a mixed sample was $2 \times 10^{7}$ cells or $2 \times 10^{6}$ cells.
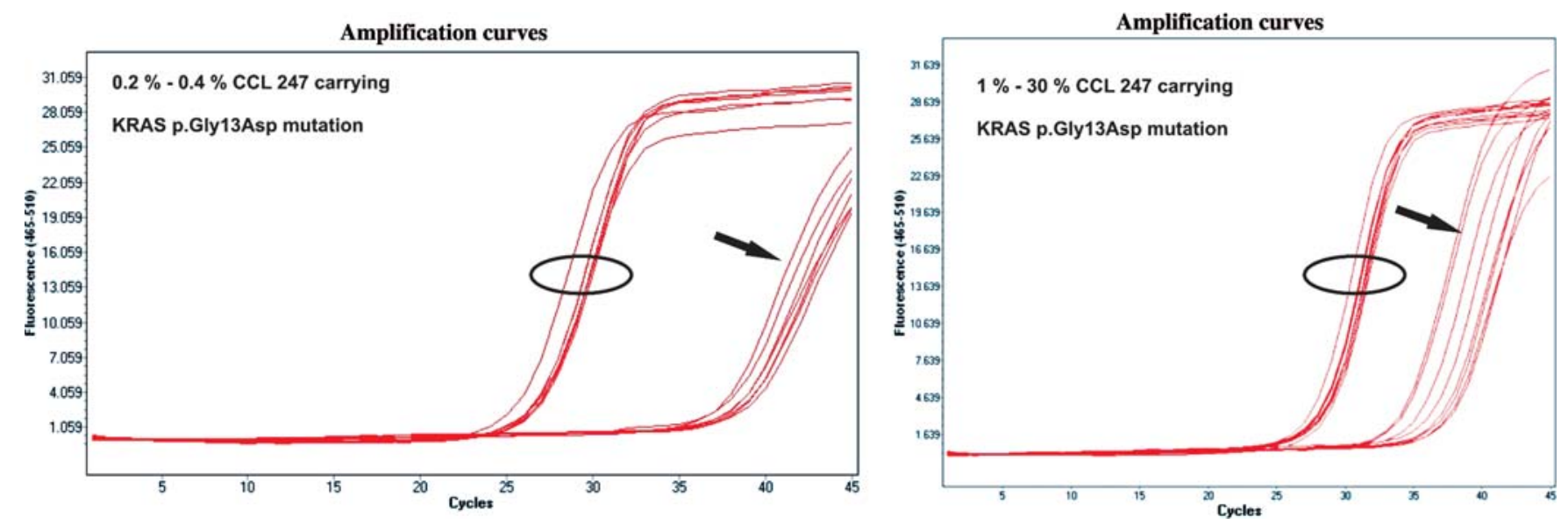

Figure 1. Amplification curves of the mixed samples harboring the p.Gly13Asp mutation of the KRAS gene. TheraSreen K-RAS Mutation Kit-amplification curves of DNA isolated from cell mixtures containing non-tumor and tumor cells harboring different proportions of cells carrying KRAS p.Gly13Asp mutation. The left picture represents amplification curves of mixed samples with $0.2-0.4 \%$ of CCL247 cells harboring the KRAS p.Gly13Asp mutation. The circled amplification curves represent the amplification of the mixed standard, while the arrow points at the group of curves representing the amplification of the KRAS p.Gly13Asp mutation. As the percentage of CCL247 tumor cells in the sample increases from 1-30\% steeper amplification curves and lower threshold cycles are observed (right picture). The circled amplification curves represent the amplification of the mixed standard, and the arrow points at the group of curves representing the amplification of the KRAS p.Gly13Asp mutation.

KRAS mutational status and the distribution of KRAS mutations. For the diagnostic purposes a group of 302 samples from 273 patients with metastatic colorectal cancer, treated at the Institute of Oncology Ljubljana, were tested. The genotyping of the KRAS gene was not possible for five patients $(1.8 \%)$ due to poor DNA quality. The test was successfully performed on $98.1 \%$ of the tested patients.

Among the successfully tested patients are 146 (54.5\%) patients with wt-KRAS genotype and $122(45.5 \%)$ patients carrying one of the tested KRAS mutation. The proportional 
Table III. The assessment of mutational status for prognostication of the cetuximab therapy outcomes in colorectal cancer patients.

\begin{tabular}{|c|c|c|c|}
\hline Sample no. & Sample ID & KRAS-status & Response to treatment \\
\hline 1 & KR5 & wt & Complete response \\
\hline 2 & KR12 & wt & Partial response \\
\hline 3 & KR10 & wt & Partial response \\
\hline 4 & KR23 & wt & Partial response \\
\hline 5 & KR22 & wt & Partial response \\
\hline 6 & KR18 & wt & Partial response \\
\hline 7 & KR21 & wt & Partial response \\
\hline 8 & KR1 & wt & Partial response \\
\hline 9 & KR7 & $12 \mathrm{Ser}$ & Partial response \\
\hline 10 & KR9 & wt & Stable disease \\
\hline 11 & $\mathrm{KR} 2$ & wt & Stable disease \\
\hline 12 & KR16 & wt & Stable disease \\
\hline 13 & KR20 & 12Arg & Stable disease \\
\hline 14 & KR25 & 12Asp & Stable disease \\
\hline 15 & KR14 & $12 \mathrm{Asp}$ & Stable disease \\
\hline 16 & KR4 & $12 \mathrm{Ser}$ & Stable disease \\
\hline 17 & KR24 & 13Asp & Stable disease \\
\hline 18 & KR19 & 13Asp & Stable disease \\
\hline 19 & KR17 & wt & Progress of disease \\
\hline 20 & KR15 & wt & Progress of disease \\
\hline 21 & KR3 & $12 \mathrm{Asp}$ & Progress of disease \\
\hline 22 & KR6 & 12Asp & Progress of disease \\
\hline 23 & KR13 & $12 \mathrm{Asp}$ & Progress of disease \\
\hline 24 & KR11 & 13Asp & Progress of disease \\
\hline 25 & KR8 & $12 \mathrm{Val}$ & Progress of disease \\
\hline
\end{tabular}

\section{Distribution of KRAS mutations}

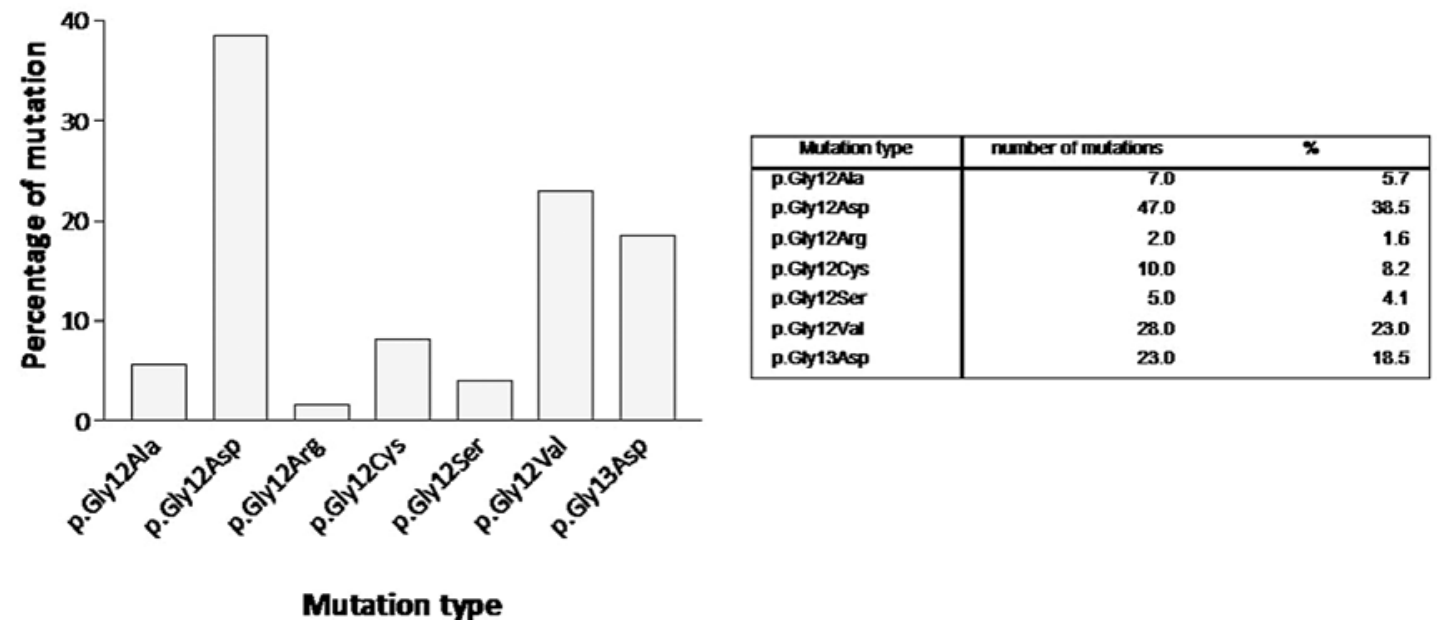

Figure 2. Distribution chart of the seven tested KRAS mutations in the group of tested patients. The proportional distribution of the seven tested KRAS mutations among the 122 positive patients.

distribution of the seven tested $K R A S$ mutations is presented in Fig. 2.
For 23 patients we obtained and tested a set of two samples; primary tumor tissue sample and metastatic tissue 
sample. From these series we were not able to perform the testing for one patient due to the poor quality of the DNA samples; primary tumor and metastatic tissue sample. Of the 22 successfully tested patients 21 patients $(95.35 \%)$ had matching results between the primary tumor tissue sample and the metastatic tissue sample. Ten $(45.5 \%)$ of the tested pair samples had a wt-KRAS genotype and $12(55.5 \%)$ of the tested pair samples were $K R A S$ mutation positive.

KRAS mutational status as a prognosticator of cetuximab therapy outcome. To asses the power of mutational status for prognostication of the cetuximab therapy outcomes we retrospectively tested the DNA samples from 23 patients with metastatic colorectal cancer. KRAS status was not known before starting and during treatment. It was determined in December 2008, retrospectively. We tested 25 samples altogether as 2 tumor samples were available for two patients. The patients were treated with cetuximab from 2 to 18 months. In one $(1 / 23)$ patient complete response was observed. With 6 patients we observed partial response (for two patients two tumor samples were available), 9 patients have stable disease and 7 patients have progressive disease. Six out of seven responders $(85.7 \%)$ were KRAS wt and 5 out of $7(71.4 \%)$ with progressive disease were KRAS mutants (Table III). One responder was observed among the 12 patients with a KRAS mutation, compared with 6 responders among the 11 patients without KRAS mutation (8.3 vs $54.5 \%$, respectively). The observed p-value from the Pearson's Chisquare test was 0.016 , which is a statistically significant difference.

\section{Discussion}

$K R A S$ status (wild-type or mutated-type) has been proven as a predictor of response to EGFR-targeted therapies for patients with metastatic colorectal cancer (10-14). The KRAS mutations are responsible for the synthesis of permanently active KRAS protein (23) and are mostly concentrated on 12 and 13 codon of the gene (17). Since activating mutations in the KRAS gene are found in $30-40 \%$ (17) of colorectal tumors, our laboratory has introduced an RT-PCR-based method (CE-marked diagnostic kit TheraScreen K-RAS Mutation Kit) to identify patients suitable for therapy with anti-EGFR inhibitors.

The assay is created to allow determination of the 7 most frequent mutations in codons 12 and 13 of KRAS gene. By the manufacturer's description, the detection level of the assay is $1 \%$ of mutated DNA in a background of wild-type DNA (25). We are performing the test in accordance with the recommendations of the European quality assurance program. This includes the identification of the patient (with the indication for testing and patient-specific medical data) by a healthcare professional, data on type of material used for testing and content of tumor cells present in the sample. The used test should be highly sensitive and specific and interpretation of results in the context of the indication for testing (17).

Before the routine daily usage of the TheraScreen K-RAS Mutation Kit for diagnostic purpose, we evaluated the sensitivity and specificity of the method in our laboratory. Firstly we tested the sensitivity of the TheraScreen K-RAS Mutation Kit using different proportions of tumor cells in the sample, to establish the minimum percentage of tumor cells carrying a mutation in a background of non-tumor cells that are required for successful mutation detection. We find this very important, as tumor samples have different amounts of tumor cells present in the sample, especially when the samples are taken after chemotherapy or irradiation therapy. This happens rarely, but the results of the testing must be interpreted even in these cases correctly. From a series of cell mixture samples that was prepared to mimic the heterogeneous tumor tissue, we determined that $2 \%$ cells carrying a KRAS mutation must be present in the sample for an undisputable detection of mutated signal using the LightCycler Adapt Software. The very same results were obtained when DNA samples with different dilutions were prepared; $2 \%$ of mutated DNA was the limit for a positive mutation call by the LightCycler Adapt Software. Our laboratory established detection limit for the method using the LightCycler Adapt Software is 2\% of mutated cells. This is to a small extent different from the detection limit declared by the manufacturer; approximately $1 \%$ of mutated DNA in the background of KRAS wt DNA (25). Indeed, if the Light Cycler Adapt software was not used and the analysis was performed manually the detection limit was increased to $0.2 \%$ of mutated cells. The possibility of an error with these dilutions is very high, if we consider the minute concentrations of the cells carrying the KRAS mutation that are present in the sample. Therefore, the difference in our laboratory established detection limit of $2 \%$ and the manufacturer's detection limit of $1 \%$ with the sensitivity interval of $1-2 \%$ is practically the same.

To assess the specificity of the method, we evaluated the concordance of our test results for 30 retrospective samples with the results of the pyrosequencing. The pyrosequencing of our samples was performed by the appointed reference laboratory at the Institute of Pathology of the LMU Munich. Our real-time PCR results were in $100 \%$ concordance when compared with the pyrosequencing method. Based on this, we gained the $K R A S$-expert laboratory certificate for quality assurance of the molecular-pathological detection of KRAS mutations in colorectal cancer in March 2009.

Among the 273 routinely-tested Slovenian patients the ratio of mutated $(45.5 \%)$ and non-mutated $(54.5 \%) \mathrm{KRAS}$ status is slightly elevated compared with the ratio reported by other European countries $(10,17,19,20,26-28)$, but will probably decrease in the favor of non-mutated KRAS as the number of tested patients increases with time. The distribution of the seven tested mutations (p.Gly12Ala $=5.7 \%$, p.Gly $12 \mathrm{Asp}=38.5 \%$, p.Gly $12 \mathrm{Arg}=1.6 \%$, p.Gly12Cys $=$ $8.2 \%$, pGly $12 \mathrm{Ser}=4.1 \%$, p.Gly $12 \mathrm{Val}=23.0 \%$ and p.Gly13Asp $=18.5 \%$ ) among the mutated KRAS patients is in concordance with the published data $(29,30)$.

In the group of routinely-tested patients there were 23 patients from whom primary tumor and metastatic tissues were available. This enabled us to investigate the correlation between KRAS mutation status of primary tumors and related metastatic tissues. Among the 22 successfully tested pair-matched samples, $21(95.35 \%)$ had a matching result between the primary tumor tissue and related metastatic tissue. We believe the discrepancy between the primary tumor 
tissue and the related metastatic tissue in the KRAS status could be attributed to the insufficient number of tumor cells in the sample, or lower method sensitivity. Ten of the tested pair samples $(45.5 \%)$ had a wt-KRAS genotype and 12 of the tested pair samples $(55.5 \%)$ were $K R A S$ mutation positive. In contrast to EGFR expression which may differ in primary colorectal tumor tissue and the corresponding metastatic tissue, literature reports high concordance between KRAS mutations of primary and metastatic tumors from patients with colorectal carcinoma $(31,32)$. This grade of concordance is important from two aspects. Firstly it suggests that KRAS mutations are an early event in colorectal carcinoma pathogenesis and are not essential for metastatic spread of the tumor (31). Secondly, if the primary tumor sample is not available, the evaluation of KRAS mutational status can also be performed from a metastatic site with relatively high confidence.

To assess the power of mutational status for prognostication of the anti-EGFR therapy outcomes, we retrospectively tested 23 patients with metastatic colorectal cancer, who were treated with cetuximab from 2 to 18 months. Out of 23 patients 7 patients responded to the therapy. As expected $85.7 \%$ of the responders were $K R A S$-wt patients. Among the wt $K R A S$ responders a complete response was achieved only by one patient, while $5 K R A S$-wt patients showed partial response. Other authors have reported $40-60 \%$ of patients with wild-type $K R A S$ responding to the treatment (33). This suggests there are other important molecular factors of response that need to be identified. On the other hand one patient from the responders group was a KRAS mutant. He responded to all previous chemotherapies with quite a long progression-free survival time, therefore, we conclude the observed response was probably due to another line of chemotherapy, rather than to combination with cetuximab. The group of patients with stable disease gives inconclusive prediction data as the KRAS mutational status is mutation negative for $33.3 \%$ of the patients. On the other hand among 16 patients out of 23 who did not respond to therapy, 9 patients had stable disease, and 7 had a progression of disease. In the group of non-responders $71.4 \%$ of patients had a mutated KRAS genotype. This supports the conclusion that the test seems to be highly specific, suggesting complete or partial response to cetuximab is highly unlikely in the presence of a KRAS mutation.

We have shown, that the RT-PCR method our laboratory has introduced to discriminate between cetuximab therapy responders and non-responders is efficient, reliable and quickly applicable. The ratio of patients with wt-KRAS and mutated KRAS status is similar to ratios reported by other authors, so is the distribution of the 7 most common mutations among the mutated KRAS patients. Even though the number of responders among the patients with wt-KRAS was statistically significantly higher compared to patients with mutated KRAS ( $\mathrm{p}=0.016$ ), the selection of patients for treatment solely on the basis of KRAS status is not perfect. Still, the testing of $K R A S$ genotype status is useful to identify patients who will respond to cetuximab, not only for reducing the toxic effects of the therapy for those patients who will not benefit from it, but also to reduce high health care costs.

\section{References}

1. Harari PM, Allen GW and Bonner JA: Biology of interactions: anti-epidermal growth factor receptor agents. J Clin Oncol 25: 4057-4065, 2007.

2. Hemming AW, Davis NL, Kluftinger A, Robinson B, Quenville NF, Liseman B and Lcriche J: Prognostic markers of colorectal cancer: an evaluation of DNA content, epidermal growth factor receptor, and Ki-67. J Surg Oncol 51: 147-152, 1992.

3. Kluftinger AM, Robinson BW, Quenville NF, Finley RJ and Davies NJ: Correlation of epidermal growth factor receptor and c-erbB2 oncogene product to known prognostic indicators of colorectal cancer. Surg Oncol 1: 97-105, 1992.

4. Mayer A, Takimoto M, Fritz E, Schellander G, Kofler K and Ludwig $\mathrm{H}$ : The prognostic significance of proliferating cell nuclear antigen, epidermal growth factor receptor, and mdr gene expression in colorectal cancer. Cancer 71: 2454-2460, 1993.

5. Salomon DS, Brandt R, Ciardiello F and Normanno N: Epidermal growth factor-related peptides and their receptors in human malignancies. Crit Rev Oncol Hematol 19: 183-232, 1995.

6. Spano JP, Lagorce C, Atlan D, et al: Impact of EGFR expression on colorectal cancer patient prognosis and survival. Ann Oncol 16: 102-108, 2005.

7. Bonomi PD, Buckingham L and Coon J: Selecting patients for treatment with epidermal growth factor tyrosine kinase inhibitors. Clin Cancer Res 13: s4606-s4612, 2007.

8. Rivera F, Vega-Villegas ME, Lopez-Brea MF and Marquez R: Current situation of panitumumab, matuzumab, nimotuzumab and zalutuzumab. Acta Oncol 47: 9-19, 2008.

9. Dassonville O, Bozec A, Fischel JL and Milano G: EGFR targeting therapies: monoclonal antibodies versus tyrosine kinase inhibitors. Similarities and differences. Crit Rev Oncol Hematol 62: 53-61, 2007.

10. Amado RG, Wolf M, Peeters M, et al: Wild-type KRAS is required for panitumumab efficacy in patients with metastatic colorectal cancer. Cancer J Clin Oncol 26: 1626-1634, 2008.

11. Kimura H, Sakai K, Arao T, Shimoyama T, Tamura T and Nishio K: Antibody-dependent cellular cytotoxicity of cetuximab against tumor cells with wild-type or mutant epidermal growth factor receptor. Cancer Sci 98: 1275-1280, 2007.

12. Lievre A, Bachet JB, Le Corre D, et al: KRAS mutation status is predictive of response to cetuximab therapy in colorectal cancer. Cancer Res 66: 3992-3995, 2006.

13. Lievre A, Bachet JB, Boige V, et al: KRAS mutations as an independent prognostic factor in patients with advanced colorectal cancer treated with cetuximab. J Clin Oncol 26: 374-379, 2008.

14. De Roock W, Piessevaux H, De Schutter J, et al: KRAS mutation status and early radiological response predict survival in colorectal cancer treated with cetuximab. Ann Oncol 19: 508515,2008 .

15. Vetter IR and Wittinghofer A: The guanine nucleotide-binding switch in three dimensions. Science 294: 1299-1304, 2001.

16. Wennerberg K, Rossman KL and Der CJ: The Ras superfamily at a glance. J Cell Sci 118: 843-846, 2005.

17. Van Krieken JHJM, Jung A, Kirchner T, et al: KRAS mutation testing for predicting response to anti-EGFR therapy for colorectal carcinoma: proposal for a European quality assurance program. Virchows Arch 453: 417-431, 2008.

18. Kiaris H and Spandidos DA: Mutations of ras genes in human tumors (Review). Int J Oncol 7: 413-421, 1995.

19. Glarakis IS, Savva S and Spandidos DA: Activation of the ras genes in malignant and premalignant colorectal tumors. Oncol Rep 5: 1451-1454, 1998.

20. Andreyev HJ, Norman AR, Cunningham D, et al: Kirsten ras mutations in patients with colorectal cancer: the 'RASCAL II' study. Br J Cancer 85: 692-696, 2001.

21. Keller JW, Franklin JL, Graves-Deal R, Friedman DB, Whitwell CV and Coffey RJ: Oncogenic KRAS provides a uniquely powerful and variable oncogenic contribution among RAS family members in the colonic epithelium. J Cell Physiol 210: 740-749, 2007.

22. Wang JY, Wang YH, Jao SW, et al: Molecular mechanisms underlying the tumorigenesis of colorectal adenomas: correlation to activated K-ras oncogene. Oncol Rep 16: 1245-1252, 2006.

23. Zenker M, Lehmann K, Schulz AL, et al: Expansion of the genotypic and phenotypic spectrum in patients with KRAS germline mutations. J Med Genet 44: 131-135, 2007. 
24. Cross J: Company Profile: DxS Ltd. Pharmacogenomics 9: 463-467, 2008

25. DxS Limited: TheraScreen KRAS mutation Kit: Instructions for use. 2009.

26. Bos JL, Fearon ER, Hamilton ER, Verlaan-de Vries M, van Boom JH, van der Eb AJ and Vogelstein B: Prevalence of ras mutations in human colorectal cancers. Nature 327: 293-297, 1987

27. Vogelstein B and Kinzler KW: Cancer genes and the pathways they control. Nat Med 10: 789-799, 2004.

28. Di Fiore F, Blanchard F, Charbonnier F, et al: Clinica relevance of KRAS mutation detection in metastatic colorectal cancer treated by cetuximab plus chemotherapy. Br J Cancer 96: 1166-1169, 2007.

29. Gnanasampanthan G, Elsaleh H, McCaul K and Iacopetta B $\mathrm{Ki}$-ras mutation type and the survival benefit from adjuvant chemotherapy in Dukes' C colorectal cancer. J Pathol 195: $543-548,2001$
30. Neumann J, Zeindl-Eberhart E, Kirchner T and Jung A: Frequency and type of KRAS mutations in routine diagnostic analysis of metastatic colorectal cancer. Pathol Res Pract 205: 858-862, 2009.

31. Santini D, Loupakis F and Vincenzi B: High concordance of KRAS status between primary colorectal tumors and related metastatic sites: implications for clinical practise. Oncologist 13: $1270-1275,2008$.

32. Artale S, Sartore-Bianchi A and Veronese SM: Mutations of KRAS and BRAF in primary and matched metastatic sites of colorectal cancer. J Clin Oncol 26: 4217-4219, 2008.

33. Linardou H, Dahabreh IJ, Kanaloupiti D, et al: Assessment of somatic K-RAS mutations as a mechanism associated with resistance to EGFR-targeted agents: a systematic review and meta-analysis of studies in advanced non-small lung cancer and metastatic colorectal cancer. Lancet Oncol 9: 962-972, 2008. 